\title{
Nanomechanical-resonator-induced synchronization in Josephson junction arrays
}

\author{
B. R. Trees* and S. Natu \\ Department of Physics and Astronomy, Ohio Wesleyan University, Delaware, Ohio 43015, USA \\ D. Stroud \\ Department of Physics, The Ohio State University, Columbus, Ohio 43210, USA
}

(Received 3 September 2005; published 30 December 2005)

\begin{abstract}
We show that a serial array of $N$ nonuniform, underdamped Josephson junctions coupled piezoelectrically to a nanoelectromechanical (NEM) oscillator results in phase locking of the junctions. Our approach is based on a semiclassical solution to a set of coupled differential equations that were generated by the Heisenberg operator equations, which in turn are based on a model Hamiltonian that includes the following effects: the charging and Josephson energies of the junctions, dissipation in the junctions, the effect of a dc bias current, an undamped simple harmonic oscillator (representing the NEM), and an interaction energy (due to the piezoelectric effect) between the NEM and the junctions. Phase locking of the junctions is signaled by a step in the current-voltage $(I-V)$ curve. We find the phase-locked states are (neutrally) stable at the bottom and top of the step but not for bias currents in the middle of the step. Using harmonic balance, we are able to calculate an analytical expression for the location of the resonance step, $v_{\text {step }}$, in the $I-V$ curve. Because of the multistability of the underdamped junctions, it is possible, with a judicious choice of initial conditions and bias current, to set a desired number $N_{a} \leqslant N$ of junctions on the resonance step, with $N-N_{a}$ junctions in the zero-voltage state. We are also able to show that, when $N_{a}$ junctions are in the phase-locked configuration, the time-averaged energy of the NEM oscillator scales like $N_{a}^{2}$.
\end{abstract}

DOI: 10.1103/PhysRevB.72.214524

PACS number(s): 74.81.Fa, 05.45.Xt, 85.85.+j

\section{INTRODUCTION}

Interest in nanoelectromechanical (NEM) structures and devices has grown rapidly over the last few years. ${ }^{1}$ From detecting microscopic mechanical displacements at near the limit set by the Heisenberg uncertainty principle ${ }^{2,3}$ to quantum information processing and entanglement, ${ }^{4-9}$ concepts at the heart of quantum computation, NEMs show promise as scientific and technological tools. It is worth noting that much of the work on quantum computation involving NEMs has focused on some kind of coupling of a NEM oscillator to a Josephson-junction (JJ) device. ${ }^{10}$ In fact, recent theoretical work by Cleland and Geller ${ }^{6}$ has demonstrated that a high$Q\left(\approx 10^{3}\right)$, high-frequency $(10 \mathrm{GHz})$, dilatational resonator made from the piezoelectric crystalline material $\mathrm{AlN},{ }^{11}$ coupled to a JJ phase qubit could be used to produce entangled states of two or more qubits. In their work, Cleland and Geller argue that although multiqubit operations by means of junction coupling through electromagnetic or superconducting resonators have been proposed, ${ }^{12}$ a mechanical resonator has the advantage of higher $Q$ values, smaller dimensions, and thus a greater potential for scalability.

In their own right, arrays of JJs biased in the nonzero voltage state have been studied extensively as systems of coupled, nonlinear oscillators. ${ }^{13-16}$ As test beds for both theoretical and experimental studies of synchronization of disordered oscillator systems, JJ arrays serve an important purpose, but in addition there has been interest for some time in arrays as sources of coherent microwave radiation. ${ }^{17-30}$ The experiments by Barbara and co-workers ${ }^{31}$ demonstrated that two-dimensional, underdamped arrays in a high- $Q$ resonance cavity formed by the array itself and a ground plane could emit coherent mm-wavelength radiation at power levels much higher than seen before. The main criteria for the high detected ac power output was a sharp resonance of the cavity and a coupling mechanism between the cavity and the individual JJs. Subsequent theoretical work by Almaas and Stroud $^{32,33}$ resulted in dynamical equations for JJ arrays coupled to a resonant cavity that reproduced well the experimental data of Barbara and co-workers. In this model, Almaas and Stroud showed that the effective coupling resulting in the coherent emission of radiation could be thought of as a capacitive coupling between the charge variables of the junctions and the cavity. Interestingly, solutions of their dynamical equations were obtained in a semiclassical limit of the Heisenberg equations for a fully quantum mechanical model Hamiltonian. This corresponds to large number of photons in the cavity and a large number of charges in the junctions, and so their results implied that much of the data of Barbara and co-workers could be explained by an essentially classical model.

In this work, we demonstrate that resonance between a serial array of underdamped JJs and a NEM oscillator can, like an electrical or superconducting cavity, result in synchronous behavior of the array. Even more so than in the case of electrical or superconducting cavities, however, this work is motivated by the possibility of attaining remarkably high- $Q$ values for AlN oscillators, ${ }^{34}$ thereby markedly increasing the likelihood of a strong resonance between oscillator and array and thus increasing the likelihood of coherent voltage outputs from the junctions in the array. In addition, modern fabrication techniques allow for NEMs and JJ arrays to be manufactured on the same chip, ${ }^{6}$ a fact which could result in smaller dimensions and increased scalability. Our work is based on an approach similar to that of Ref. 32. We 
construct a Hamiltonian describing a serial array of $N$ underdamped JJs coupled to a dilatational AlN oscillator. The coupling between the oscillator and the array is electrical by means of the piezoelectric nature of AlN. Both the electrostatic charging energy as well as the Josephson energy are included in the Hamiltonian for the junctions. Damping in the junctions is included via the Caldeira-Leggett approach of coupling each junction to a bath of independent harmonic oscillators. ${ }^{35}$ We ignore damping in the NEM oscillator itself, corresponding to the limit $Q \rightarrow \infty$, which is not unreasonable given the possibility of high- $Q$ factors in AlN oscillators as mentioned above. It would not be difficult, however, to add oscillator damping. Heisenberg equations generate the dynamical equations of motion for this system, which are then solved in the semiclassical limit, corresponding to a large number of NEM phonons and large number of charges in the junctions. The oscillator-array resonance is manifested by a step in the current-voltage $(I-V)$ curve of the array. We demonstrate the existence of such a step and show that it is possible, using the hysteretical property of the junctions, to bias $N_{a} \leqslant N$ (active) junctions on the step by an appropriate choice of initial conditions. We also perform a linear stability analysis of the coherent array solutions and find that the coherence is only neutrally stable at the ends of the step, i.e., that, surprisingly, there is a region in the middle of the step where the coherent behavior of the array is unstable. We are able to solve analytically for the voltage $v_{\text {step }}$ at which the step occurs, and we show that the time-averaged NEM energy, when in resonance with the junctions and the junctions are phase locked, scales like the square of the number of active junctions. In our model, for sufficiently large coupling between the array and the NEM oscillator, the frequency of the NEM oscillator can be renormalized upward, so that the resonance voltage, $v_{\text {step }}$, may not be at the location expected based on the NEMs natural, i.e., uncoupled, frequency. We add that the Hamiltonian describing this system could in principle be used for a full quantum-mechanical treatment of such a system. With proper biasing of the junctions, such a treatment may be relevant to an understanding of coupled multiqubit systems.

This paper is organized as follows. In Sec. II, we construct the classical Hamiltonian describing a single junction coupled to the AlN oscillator. We generate classical equations of motion for the system and briefly discuss their physical significance. In Sec. III, we construct the classical Hamiltonian for a serial array of JJs coupled to a single AIN oscillator. The junctions have nonuniform critical currents and so would naturally produce different time-average voltages if uncoupled or only weakly coupled. We then construct the quantum Hamiltonian for the serial array plus oscillator and generate the Heisenberg equations of motion. In Sec. IV, we study the numerical solutions of the equations of motion in the semiclassical limit, showing the resonance step as evidence for coherent behavior of the junctions. We also have performed a stability analysis which shows that neutrally stable synchronization, i.e. coherence, occurs only at the two edges of the step. The Kuramoto order parameter, which measures the degree of phase coherence of the junctions, is also calculated and, like the stability analysis, shows coherent behavior only at the two edges of the step. In Sec. V, we

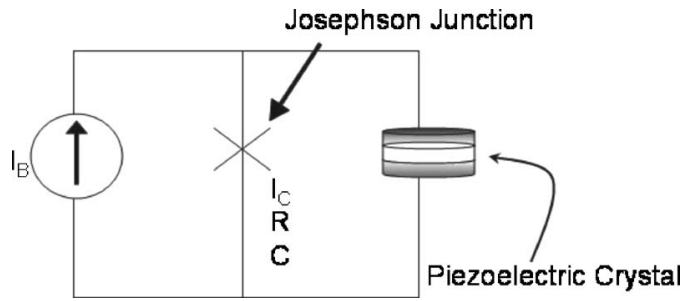

FIG. 1. A single Josephson junction and a dc current source $I_{B}$ in parallel with a NEM oscillator. The junction has critical current $I_{c}$, capacitance $C$, and shunt resistance $R$. The oscillator consists of a piezoelectric crystal sandwiched between the plates of a capacitor. The effective capacitance of the plates and crystal is $C_{o}$. The mechanical strain of the crystal, denoted by $U$, is taken to be along the $z$ axis and in a direction perpendicular to the disk.

derive an expression for the location of the voltage step, $v_{\text {step }}$, based on the equations derived in Sec. III. We are also able to calculate analytically the average energy of the AlN oscillator while in resonance with the array; the result shows that the time-averaged oscillator energy scales like $N_{a}^{2}$, i.e., like the number of active junctions (junctions biased on the resonance step) squared. In Sec. VI, we discuss our results and conclude with directions for future work.

\section{SINGLE JUNCTION PLUS OSCILLATOR}

We start with a single JJ in parallel with a dilatational, piezoelectric NEM oscillator as described in Ref. 6 and as shown in Fig. 1. To ensure we have a reasonable classical Hamiltonian for this system, we start by constructing a Lagrangian from which the classical equations of motion give expected results. The Lagrangian describing the system is taken to be

$$
L(\gamma, U, \dot{\gamma}, \dot{U})=T_{N E M}+T_{J}-U_{N E M}-U_{J}-U_{i n t},
$$

where there are kinetic energy and potential terms each for the oscillator and JJ, and a potential energy representing an interaction between the JJ and oscillator. The Lagrangian depends on $\gamma$, the gauge-invariant Josephson phase difference across the junction $U$, the spatially averaged strain in the piezoelectric crystal (the strain is perpendicular to the plates of the oscillator and is assumed to be in the $z$ direction), and their time derivatives $\dot{\gamma}$ and $\dot{U}$. The individual terms in Eq. (1) are as follows:

$$
T_{N E M}=\frac{M_{N E M} \dot{U}^{2}}{2}
$$

where $M_{N E M}$ is an effective mass for the oscillator. Note $U$, the crystal strain, is dimensionless, and so $M_{N E M}$ has dimensions of $M \times L^{2}$ (mass times length squared).

$$
T_{J}=\frac{M_{J} \dot{\gamma}^{2}}{2},
$$

where $M_{J}=\hbar^{2}\left(C+C_{o}\right) /(2 e)^{2}$, where $C$ is the capacitance of the JJ, and $C_{o}$ the capacitance of the oscillator. The dimensions of $M_{J}$ are also $M \times L^{2}$. 


$$
U_{R}=\frac{1}{2} M_{N E M} \omega_{0}^{2} U^{2}
$$

where $\omega_{0}$ is the natural frequency of oscillation of the independent oscillator and the crystal strain $U$ plays the role of the oscillator's displacement.

$$
U_{J}=-\frac{\hbar I_{c}}{2 e}\left(\cos \gamma+\frac{I_{B}}{I_{c}} \gamma\right)
$$

where $I_{c}$ is the junction critical current and $I_{B}$ is the dc bias current. Based on Ref. 6, we use an interaction energy of the form

$$
U_{\text {int }}=\mu \gamma \dot{U},
$$

where $\mu$ is the interaction strength with dimensions of $M$ $\times L^{2} / T$. Next, we construct the Hamiltonian as the Legendre transform of the Lagrangian

$$
H\left(P_{R}, P_{J}, U, \gamma\right)=P_{R} \dot{U}+P_{J} \dot{\gamma}-L,
$$

where $P_{R}$ and $P_{J}$ are the momenta of the oscillator and junction, respectively. Using Eqs. (1)-(6), we get a Hamiltonian of the form

$$
\begin{aligned}
H= & \frac{P_{R}^{2}}{2 M_{N E M}}+\frac{1}{2} M_{N E M} \omega_{0}^{2} U^{2}+\frac{P_{J}^{2}}{2 M_{J}}+U_{J}(\gamma)+\frac{\mu^{2} \gamma^{2}}{2 M_{N E M}} \\
& +\frac{\mu \gamma P_{R}}{M_{N E M}} .
\end{aligned}
$$

Using Hamilton's equations, we generate the classical equations of motion for this coupled system. Including dissipation in the $\mathrm{JJ}$, we get

$$
\begin{gathered}
I_{c} \sin \gamma+\frac{\hbar}{2 e R} \dot{\gamma}+\frac{\hbar\left(C+C_{o}\right)}{2 e} \ddot{\gamma}+\frac{2 e M_{N E M} \lambda}{\hbar} \dot{U}=I_{B}, \\
\ddot{U}+\omega_{0}^{2} U=\lambda \dot{\gamma},
\end{gathered}
$$

where $\lambda=\mu / M_{N E M}$ is a coupling constant with dimensions of $1 / T$ and $R$ is the shunt resistance of the junction. Equations (9) and (10) seem physically reasonable for a couple of reasons. For example, these equations result when one invokes conservation of charge for a single JJ coupled to a dissipationless oscillator driven by a current source $I_{B}$ (see Fig. 1) and make use of the resistively and capacitively-shunted junction (RCSJ) model for the junction. This is based, however, upon assuming that the current through the oscillator has the form ${ }^{6}$

$$
I_{o}=C_{o}\left(\dot{V}-e_{33} b \dot{U} / \epsilon_{33}\right),
$$

where $e_{33}$ and $\epsilon_{33}$ are the appropriate elements of the piezoelectric modulus and dielectric tensors, respectively; $\dot{V}$ is the time derivative of the voltage across the $\mathrm{JJ}$; and $b$ is the diameter of the crystal. One can then learn that the coupling constant between the junction and oscillator can be written as

$$
\lambda=-\hbar C_{o} e_{33} b /\left(2 e M_{N E M} \epsilon_{33}\right) .
$$

In addition, Eqs. (9) and (10), respectively, have the same structure as those describing the $c$-axis current density and

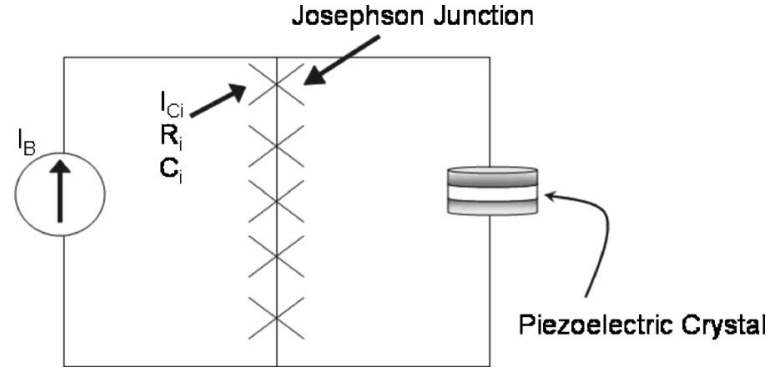

FIG. 2. A serial Josephson junction array and a dc current source $I_{B}$ in parallel with a NEM oscillator. Junction $i$ has critical current $I_{c i}$, capacitance $C_{i}$, and shunt resistance $R_{i}$. The oscillator consists of a piezoelectric crystal sandwiched between the plates of a capacitor. The effective capacitance of the plates and crystal is $C_{o}$.

polarization in the work of Helm and collaborators, ${ }^{36}$ in which the subgap structure in the $I-V$ characteristics of high$T_{c}$ cuprates was explained as a resonance between Josephson oscillations between neighboring copper-oxide planes and $c$-axis phonons.

\section{SERIAL ARRAY PLUS OSCILLATOR}

\section{A. Classical Hamiltonian}

We now generalize Eq. (1) to the case of a disordered, serial array (see Fig. 2). Based on Eq. (11), the current through the oscillator would be

$$
I_{o}=C_{o}\left[\frac{\hbar}{2 e} \sum_{k} \ddot{\gamma}_{k}-\frac{e_{33} b \dot{U}}{\epsilon_{33}}\right]=\frac{\hbar C_{o}}{2 e} \sum_{k} \ddot{\gamma}_{k}+\frac{2 e M_{m e m} \lambda}{\hbar},
$$

where the Josephson voltage across junction $k, V_{k}=\hbar \dot{\gamma}_{k} / 2 e$, was used. The last term on the right of Eq. (13) was obtained by using Eq. (12).

Conservation of charge and the RCSJ equation yield for the $j$ th junction

$$
I_{B}=I_{c j} \sin \gamma_{j}+\frac{\hbar}{2 e R_{j}} \dot{\gamma}_{j}+\frac{\hbar C_{j}}{2 e} \ddot{\gamma}_{j}+\frac{\hbar C_{o}}{2 e} \sum_{k} \ddot{\gamma}_{k}+\frac{2 e M_{N E M} \lambda}{\hbar} \dot{U}
$$

The oscillator's equation of motion would be [compare with Eq. (10)]

$$
\ddot{U}+\omega_{0}^{2} U=\lambda \sum_{k} \dot{\gamma}_{k}
$$

where the term on the right of Eq. (15) is due to the fact that the oscillator would be driven by the total voltage across the array. As can be verified directly by Lagrange's equations of motion, the Lagrangian that results in Eqs. (14) and (15) is 


$$
\begin{aligned}
L= & \sum_{j=1}^{N}\left[\frac{1}{2} M_{J j} \dot{\gamma}_{j}^{2}-U_{J j}\left(\gamma_{j}\right)\right]+\frac{1}{2} M_{N E M} \dot{U}^{2}-\frac{1}{2} M_{N E M} \omega_{0}^{2} U^{2} \\
& -\lambda M_{N E M} \dot{U} \sum_{j=1}^{N} \gamma_{j}+\frac{\hbar^{2} C_{o}}{8 e^{2}}\left(\sum_{j=1}^{N} \dot{\gamma}_{j}\right)^{2}
\end{aligned}
$$

where $M_{J j}=\hbar^{2} C_{j} /\left(4 e^{2}\right)$ is the "mass" of junction $j$ and the potential energy of junction $j$ is $U_{J j}\left(\gamma_{j}\right)=-\left[\left(\hbar I_{c j}\right) /\right.$ $2 e]\left[\cos \gamma_{j}+\left(I_{B} / I_{c j}\right) \gamma_{j}\right]$. The next step is to construct the Hamiltonian from Eq. (16). To do so, we need the momenta conjugate to the variables $U$ and $\gamma_{j}$, which are, respectively,

$$
\begin{gathered}
P_{R}=\frac{\partial L}{\partial \dot{U}}=M_{N E M} \dot{U}-\lambda M_{N E M} \sum_{k=1}^{N} \gamma_{k}, \\
P_{J j}=\frac{\partial L}{\partial \dot{\gamma}_{j}}=M_{J j} \dot{\gamma}_{j}+\frac{\hbar^{2} C_{o}}{4 e^{2}} \sum_{k=1}^{N} \dot{\gamma}_{k} .
\end{gathered}
$$

For small amounts of disorder, it should be reasonable to make the substitution $M_{J j} \rightarrow\left\langle M_{J}\right\rangle$, where $\left\langle M_{J}\right\rangle$ is the arithmetic average of the junction masses, namely $\left\langle M_{J}\right\rangle$ $=\hbar^{2}\langle C\rangle / 4 e^{2}$ and $\langle C\rangle \equiv(1 / N) \sum_{j=1}^{N} C_{j}$. With this approximation, algebra yields a Hamiltonian for the serial array coupled to the oscillator of the following form:

$$
\begin{aligned}
H= & \frac{P_{R}^{2}}{2 M_{N E M}}+\frac{1}{2} M_{N E M} \omega_{0}^{2} U^{2}+\sum_{j=1}^{N}\left(\frac{P_{J j}^{2}}{2\left\langle M_{J}\right\rangle}+U_{J j}\left(\gamma_{j}\right)\right) \\
& -\frac{1}{2} \frac{\left\langle M_{J}\right\rangle}{\left(N+\frac{\langle C\rangle}{C_{o}}\right)}\left(\sum_{j=1}^{N} \frac{P_{J j}}{\left\langle M_{J}\right\rangle}\right)^{2}+\frac{\lambda^{2} M_{N E M}}{2}\left(\sum_{j=1}^{N} \gamma_{j}\right)^{2} \\
& +\lambda P_{R} \sum_{j=1}^{N} \gamma_{j} .
\end{aligned}
$$

It is useful to generate the classical equations of motion corresponding to Eq. (19). Based on $\partial H / \partial \gamma_{j}=-\dot{P}_{J j}$, we get

$$
\begin{aligned}
I_{B}= & I_{c j} \sin \gamma_{j}+\frac{\hbar\left\langle R^{-1}\right\rangle}{2 e} \dot{\gamma}_{j}+\frac{\hbar\langle C\rangle}{2 e} \ddot{\gamma}_{j}+\frac{\hbar C_{o}}{2 e} \sum_{k=1}^{N} \ddot{\gamma}_{k} \\
& +\frac{2 e \lambda M_{N E M}}{\hbar} \dot{U} .
\end{aligned}
$$

Note that we have added a dissipative term for the junctions that depends on the arithmetic average of the junction shunt resistances. Equation (20) has the form of Eq. (14) with the individual junction capacitances replaced by the arithmetic average of all $N$ junction capacitances. Based on $\partial H / \partial U=$ $-\dot{P}_{R}$, we get

$$
\ddot{U}+\omega_{0}^{2} U=\lambda \sum_{k=1}^{N} \dot{\gamma}_{k},
$$

which agrees with Eq. (15).

\section{B. Quantum Hamiltonian}

The Hamiltonian (19) can be readily quantized. We write $H=H_{J}+H_{N E M}+H_{\text {int }}+H_{\text {diss }}$, where the Hamiltonian for a serial array of junctions biased by a current source is

$$
H_{J}=\sum_{j=1}^{N}\left[\frac{2 e^{2}}{\langle C\rangle} n_{j}^{2}-\frac{\hbar I_{c j}}{2 e} \cos \gamma_{j}-\frac{\hbar I_{B}}{2 e} \gamma_{j}\right],
$$

where we have used that $\left\langle M_{J}\right\rangle=\hbar^{2}\langle C\rangle / 4 e^{2}$ and $P_{J j}$ is replaced by $\hbar n_{j}$, where $n_{j}$ is the operator representing the difference in the number of Cooper pairs between superconducting islands $j$ and $j+1$. The Hamiltonian for a NEM oscillator is taken to be that of an undamped simple harmonic oscillator of natural frequency $\omega_{0}$

$$
H_{N E M}=\hbar \omega_{0}\left(a^{\dagger} a+\frac{1}{2}\right),
$$

where the standard creation and destruction operators for the simple harmonic oscillator have been used. If we let $a \equiv a_{R}$ $+i a_{I}$ and $a^{\dagger} \equiv a_{R}-i a_{I}$, then Eq. (23) becomes

$$
H_{N E M}=\hbar \omega_{0}\left(a_{R}^{2}+a_{I}^{2}+\frac{1}{2}\right) \text {. }
$$

The dissipation in the array is handled by coupling each junction to a bath of harmonic oscillators with a specified distribution of frequencies $\omega_{\alpha}{ }^{35}$

$$
\begin{aligned}
H_{d i s s}= & \sum_{j, \alpha}\left[f_{\alpha, j} \gamma_{j} u_{\alpha, j}+\frac{p_{\alpha, j}^{2}}{2 m_{\alpha, j}}+\frac{1}{2} m_{\alpha, j} \omega_{\alpha, j}^{2} u_{\alpha, j}^{2}\right. \\
& \left.+\frac{\left(f_{\alpha, j}^{2}\right)}{2 m_{\alpha, j} \omega_{\alpha, j}^{2}} \gamma_{j}^{2}\right]
\end{aligned}
$$

where $m_{\alpha, j}$ is the mass of the $\alpha$ th oscillator connected to junction $j, u_{\alpha, j}$ is the displacement of that oscillator from equilibrium, and $f_{\alpha, j}$ represents the coupling between junction $j$ and oscillator $\alpha$. The bath oscillators are assumed to be described by a spectral density that is linear in $|\omega|$ such that the dissipation in the junctions is Ohmic. ${ }^{32,35}$ Finally, the interaction term in the Hamiltonian is based on the last three terms in Eq. (19),

$$
\begin{aligned}
H_{\text {int }}= & -\frac{2 e^{2}}{\langle C\rangle} \frac{1}{N+\frac{\langle C\rangle}{C_{o}}}\left(\sum_{j=1}^{N} n_{j}\right)^{2}+\frac{\lambda^{2} M_{N E M}}{2}\left(\sum_{j=1}^{N} \gamma_{j}\right)^{2} \\
& +2 \lambda \sqrt{\frac{\hbar M_{N E M} \omega_{0}}{2}} a_{I} \sum_{j=1}^{N} \gamma_{j} .
\end{aligned}
$$

The full Hamiltonian, given by Eqs. (22)-(26), is used to generate dynamical operator equations by means of the Heisenberg equation

$$
\frac{d O}{d t}=\frac{1}{i \hbar}[O, H],
$$

where the operator $O=n_{k}, \gamma_{k}, a_{R}$, or $a_{I}$. The following set of commutation relations are required:

$$
\left[n_{j}, \gamma_{k}\right]=-i \delta_{j, k},
$$




$$
\begin{gathered}
{\left[p_{\alpha j}, u_{\beta k}\right]=-i \hbar \delta_{\alpha, \beta} \delta_{j, k},} \\
{\left[a_{R}, a_{I}\right]=\frac{i}{2} .}
\end{gathered}
$$

We then write the resultant equations in terms of dimensionless quantities, which are listed below. Note that $\left\langle I_{c}\right\rangle$ $\equiv \sum_{j} I_{c j} / N$ and $\left\langle R^{-1}\right\rangle \equiv \Sigma_{j} R_{j}^{-1} / N$ are arithmetic averages over two key junction parameters, critical current and (inverse) shunt resistance. Also, we define a characteristic angular frequency for the junctions $\omega_{J} \equiv 2 e\left[\left\langle I_{c}\right\rangle /\left(\hbar\left\langle R^{-1}\right\rangle\right)\right]$

$$
\begin{gathered}
\tau \equiv \omega_{J} t \text { (time), } \\
i_{B} \equiv \frac{I_{B}}{\left\langle I_{c}\right\rangle} \text { (bias current), } \\
i_{c k} \equiv \frac{I_{c k}}{\left\langle I_{c}\right\rangle}(\text { critical current for junction } k), \\
\Lambda \equiv\left[\lambda \sqrt{\frac{2 e M_{N E M}}{\hbar\left\langle I_{c}\right\rangle}}\right]^{1 / 2}
\end{gathered}
$$

(coupling strength between array and oscillator),

$$
\begin{gathered}
\Omega \equiv \frac{\omega_{o}}{\omega_{J}} \text { (NEM oscillator's natural frequency) } \\
\tilde{n}_{k} \equiv \frac{4 e^{2}}{\hbar\left\langle R^{-1}\right\rangle} n_{k} \text { (number operator) } \\
\tilde{a}_{R(I)} \equiv \sqrt{\frac{e \omega_{0}}{\left\langle I_{c}\right\rangle}} a_{R(I)} \text { (oscillator operators) }
\end{gathered}
$$

The four coupled equations of motion for the dimensionless operators $\tilde{n}_{k}, \gamma_{k}, \tilde{a}_{R}$, and $\tilde{a}_{I}$ are as follows:

$$
\begin{aligned}
& \frac{d \tilde{n}_{k}}{d \tau}= i_{B}-i_{c k} \sin \gamma_{k}-\Lambda \sum_{j=1}^{N} \gamma_{j}-2 \sqrt{\Lambda} \tilde{a}_{I} \\
&-\frac{i_{c k}}{\beta_{c}}\left[\tilde{n}_{k}-\frac{1}{N\left(1+\frac{\langle C\rangle}{C_{o}}\right)} \sum_{j=1}^{N} \tilde{n}_{j}\right], \\
& \frac{d \gamma_{k}}{d \tau}= \frac{1}{\beta_{c}}\left[\begin{array}{c}
N\left(1+\frac{\langle C\rangle}{C_{o}}\right) \\
\left.\tilde{n}_{k}-\frac{1}{j\left(\tilde{n}_{j}\right.}\right],
\end{array}\right] \\
& \frac{d \tilde{a}_{R}}{d \tau}=\Omega\left[\tilde{a}_{I}+\frac{1}{2} \sqrt{\Lambda} \sum_{j=1}^{N} \gamma_{j}\right],
\end{aligned}
$$

$$
\frac{d \widetilde{a}_{I}}{d \tau}=-\Omega \widetilde{a}_{R}
$$

To obtain this set of equations, several steps were involved. The oscillator bath variables can be integrated out by wellknown techniques. ${ }^{37}$ The effect is to make the replacement

$$
\sum_{\alpha}\left[f_{\alpha, k} u_{\alpha, k}+\frac{f_{\alpha, k}^{2} \gamma_{k}}{m_{\alpha, k} \omega_{\alpha, k}^{2}}\right] \rightarrow \frac{\hbar}{2 \pi}\left(\frac{h}{4 e^{2} R_{k}}\right) \frac{d \gamma_{k}}{d t}
$$

throughout the equations of motion. Also, we make the assumption that the product $I_{c k} R_{k}$ is uniform throughout the array and that $I_{c k} R_{k}=\left\langle I_{c}\right\rangle /\left\langle R^{-1}\right\rangle$. As a result, we can make the replacement in Eq. (42)

$$
R_{k} \rightarrow \frac{\left\langle I_{c}\right\rangle}{I_{c k}\left\langle R^{-1}\right\rangle}=\frac{1}{i_{c k}\left\langle R^{-1}\right\rangle},
$$

where Eq. (33) was used in the last step.

\section{NUMERICAL RESULTS}

We solved Eqs. (38)-(41) numerically using a fourthorder Runge-Kutta algorithm with time steps $\Delta \tau=0.001$. We calculated the $I-V$ curves for several different sets of values of junction and oscillator parameters. Figure 3(a) shows a step induced by a resonance between the junctions and the NEM oscillator. The arithmetic average of the time-average voltages across the $N=10$ junctions, $\langle v\rangle_{\tau} / N$, was calculated as a function of the dc bias current $i_{B}$. An averaging interval of 250000 time steps was used to calculate $\langle v\rangle_{\tau}$ for each value of the current. The graph was produced by starting with a bias current large enough to ensure that all the junctions were in the voltage state, in this case $i_{B}=1.2$. The junction critical currents were assigned nonrandomly according to the function

$$
i_{c k}=1+\Delta-\frac{2 \Delta}{(N-1)^{2}}\left[4 k^{2}-4(N+1) k+(N+1)^{2}\right],
$$

where $\Delta=0.05$ was chosen. Such a function results in critical currents that are a parabolic function of position along the array. The $i_{c k}$ values are symmetric with respect to the center of the array. Since the junction critical currents are nonuniform, there is a spread in their time-averaged voltages. This is quantified by the error bars, which represent the standard deviation of the mean time-averaged voltage for the $N$ junctions. The bias current was reduced from its starting value. Because the junctions are underdamped $\left(\beta_{c}>1\right)$, they remain in the voltage state for $i_{B}<1$, exhibiting Ohmic behavior. The spread in the time-averaged voltages of the junctions decreases as $i_{B}$ is decreased, as demonstrated by the reduction in the size of the error bars. This is evidence of frequency synchronization of the junctions, that is, in our dimensionless system of units, frequency synchronization means all $N$ junctions have the same time-average voltage. A resonant step at $\langle v\rangle_{\tau} / N \approx \Omega$ is evident. Presumably, this step is the result of a kind of mode locking between the oscillating JJs and the AlN oscillator. This is where the coherent junction behavior is expected. 


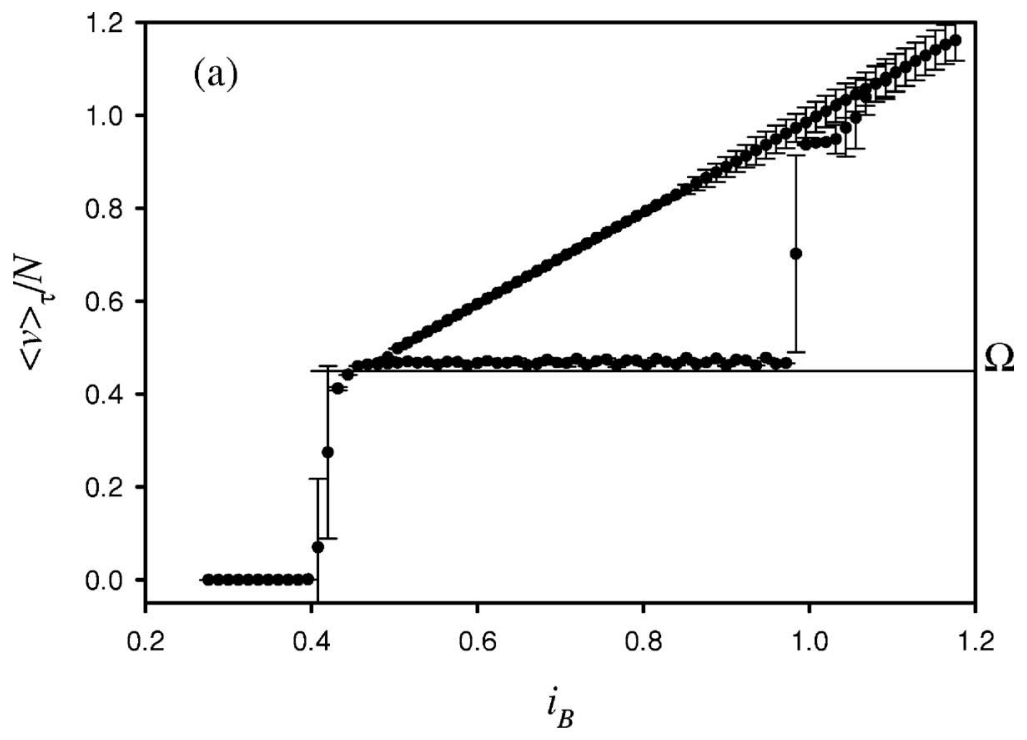

FIG. 3. (Color online) (a) $I-V$ curve for a serial array of $N=10$ underdamped junctions coupled to a NEM oscillator. Junction critical currents are assigned nonrandomly with $\Delta=0.05$ and $\beta_{c}=10$. The NEM's natural frequency, scaled by the Josephson frequency, is $\Omega=\omega_{o} / \omega_{J}=0.45$, and the coupling strength between the array and the NEM oscillator is $\Lambda=0.2$. The ratio of the average junction capacitance to the capacitance of the NEM is $\langle C\rangle / C_{o}=1$. A step due to resonance between the junctions and the NEM is evident at a voltage only slightly greater than $\Omega$, namely $\langle v\rangle_{\tau} / N=0.4683 \pm 0.0007$. The error bars on the symbols represent the standard deviation of the mean of the time-averaged JJ voltages. As the bias current is decreased from its starting value of $i_{B}=1.2$, the error bars become smaller than the symbol size, signaling approximate frequency synchronization of the array. (b) Magnitude of the ten largest Floquet multipliers corresponding to on-step solutions for the same system parameters as in (a). For the stability analysis, the junction parameters were taken to be uniform, and $|\mu|$ was calculated as the bias current was ramped up while the system was on the step. For bias currents in the middle of the step, the system is linearly unstable. Near the top of the step, $i_{B}$ $>0.85$, there is neutrally stable synchronization with two multipliers of magnitude unity. The next smallest multiplier has a magnitude reduced by 3 to $5 \%$ from unity. The system may also be neutrally stable in a very narrow bias current range at the bottom of the step.

$i_{B}$

Interestingly, a stability analysis [see Fig. 3(b)] shows that the solutions to Eqs. (38)-(41) are linearly unstable over a large fraction of the resonance step. Only for larger bias currents near the right edge ("top") of the step, and possibly for a very narrow region at the left edge ("bottom") of the step, do we see neutrally stable synchronization of the junctions and resonator. This behavior is not necessarily surprising, however, given the discovery by Watanabe and co-workers ${ }^{16}$ of parametric instabilities (demonstrated by steps in the $I-V$ curve) in Josephson rings. They found complicated dynamical behavior of the junctions that showed sensitive dependence on the location of the bias current on the step. For the $\mathrm{JJ}$ rings, they found that stable, in-phase solutions occurred only near the bottom and top of a given step. We find neutrally stable, in-phase solutions near the top of the step and possibly along a very narrow region at the bottom of the step. Our linear stability analysis was based on a numerical calculation of the Floquet multipliers $\mu$ of the solutions to Eqs. (38)-(41). ${ }^{38}$ Our system has $2 N+2$ degrees of freedom, and so the stability will be described by $2 N+2$ multipliers. The solution is unstable if any of the (generally complex) multipliers has a magnitude greater than unity. Neutral sta- bility of degree $\nu$ is signaled by $\nu+1$ multipliers of magnitude unity. Stable solutions result in all but one multiplier with magnitude less than unity. (There will always be one multiplier of magnitude unity corresponding to a perturbation tangent to the trajectory in phase space.)

We have also calculated the Kuramoto phasesynchronization order parameter $r(\tau)$ both on the resonance step as well as on the Ohmic branch

$$
r(\tau) \equiv \frac{1}{N} \sum_{k=1}^{N} e^{i \gamma_{k}(\tau)} .
$$

Figure 4(a) shows the time-averaged modulus of the order parameter, $r=\langle|r(\tau)|\rangle_{\tau}$, for decreasing bias currents on the Ohmic branch, while Fig. 4(b) corresponds to increasing bias currents along the step. Just as Fig. 3(a) shows evidence of frequency synchronization as the bias current is decreased along the Ohmic branch, we see here evidence of phase synchronization as well. That is, as $i_{B}$ is decreased along the Ohmic branch, $r \rightarrow 1$. On the step, phase synchronization is evident mainly near the top of the step, with the bias current values for which $r \approx 1$ corresponding roughly to the region of 

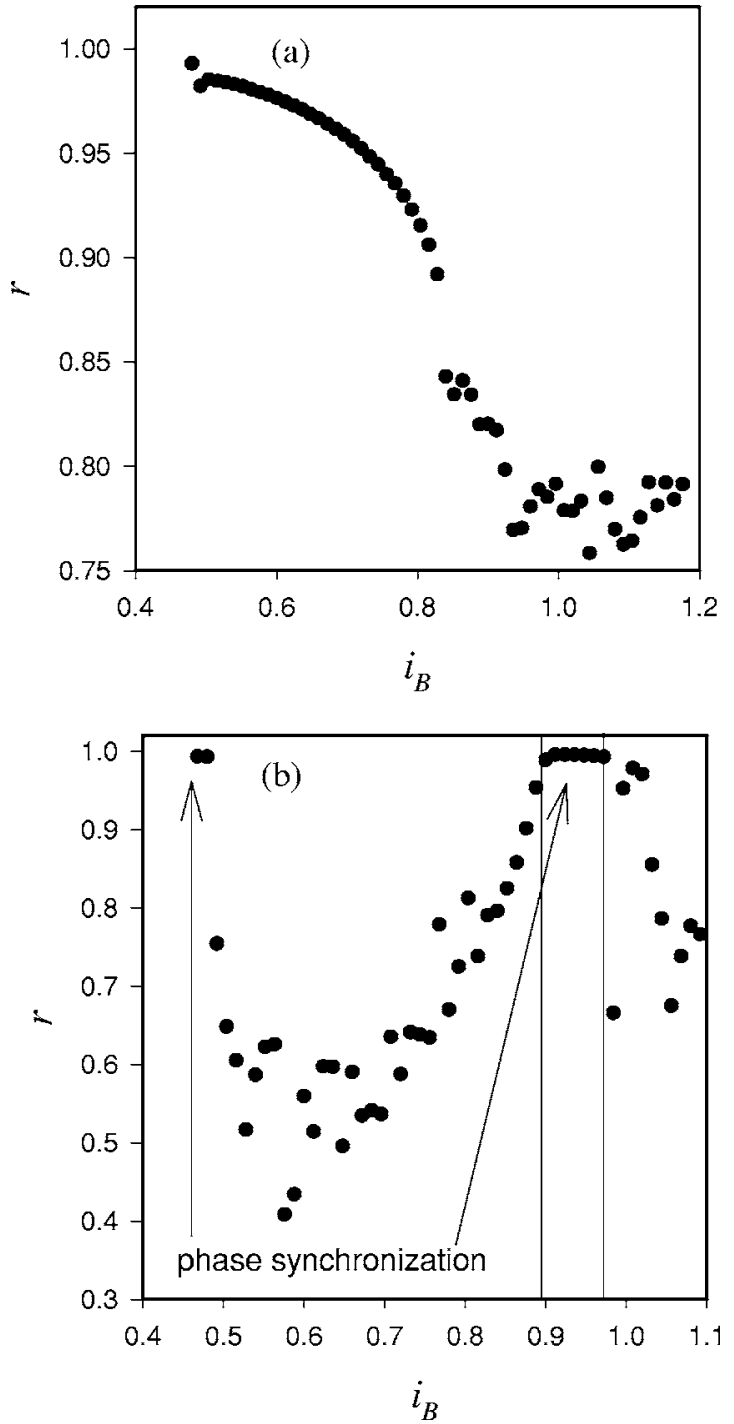

FIG. 4. Time average of the Kuramoto order parameter's modulus for a serial array of $N=10$ junctions $\left(\beta_{c}=10\right.$ and nonrandomly assigned critical currents with $\Delta=0.05$ ) coupled to a NEM oscillator with scaled natural frequency $\Omega=0.45$. The coupling strength between the array and the NEM oscillator is $\Lambda=0.2$ and $\langle C\rangle / C_{o}=1$. (a) The bias current is decreased from a starting value of $i_{B}=1.2$. The array approaches phase synchronization, $r \rightarrow 1$, as $i_{B}$ is decreased toward the step. (b) Kuramoto order parameter versus bias current as $i_{B}$ is increased along the step. Phase synchronization is evident along a very narrow region at the bottom of the step as well as along a broader region at the top of the step.

neutral stability shown in Fig. 3(b). There is also a small range of bias currents at the bottom of the step for which phase synchronization is evident.

It is useful to show the dynamic behavior of the junctions and the NEM oscillator for biasing both on the Ohmic branch and the resonant step. This is shown in Fig. 5, where in part (a) of the figure, the displacement of the oscillator, which is proportional to $\widetilde{a}_{R}$, is plotted versus dimensionless time for $i_{B}=0.9$ both on the Ohmic branch and on the resonance step. Note the different scales on the two vertical axes. On the resonance step, the amplitude of the oscillator's dis- placement is roughly 2000 times its value on the Ohmic branch at the same bias current. Also, a rough estimate based on the plots shows that the angular frequency of the oscillator's displacement is about $\Omega=0.45$, its natural value, when on the Ohmic branch, but when in resonance, it is about 0.465 , which is close to the step voltage of 0.468 . In Fig. 5 (b), the time dependence of the voltage across junction 1 is shown both off and on the step for $i_{B}=0.9$. Again, note the different scales on the two vertical axes. On the step, the voltage $v_{1}$ has the same angular frequency as $\tilde{a}_{R}$ but is $\pi$ radians out of phase with $\tilde{a}_{R}$. The behavior of $v_{1}(\tau)$ is quite complicated off the step, but if the angular frequency of the oscillations near $\tau=400$ is estimated, one gets a value of 0.93 , close to $i_{B}=0.9$. Also, it is clear that the amplitude of the voltage oscillations is significantly greater when the junction is biased on the step.

We have studied the $I-V$ characteristics of the array for larger coupling strengths $\Lambda$. We find that as the coupling to the NEM oscillator increases the step voltage, $v_{\text {step }}$, shifts to higher values and the width of the flat portion of the step decreases (see Fig. 6). Also, the retrapping current, at which the junctions begin to drop into the zero-voltage state, occurs at a value roughly equal to $\Omega$, the bare NEM oscillator frequency, in our dimensionless system of units. Figure 7 shows the step voltage as a function of the coupling between the array and oscillator. The step voltage deviates from $\Omega$ for $\Lambda>0.1$. Evidently, for strong coupling, the resonance frequency is renormalized upward. The solid line in Fig. 7 will be discussed in Sec. V.

We have checked that the same type of behavior occurs for other array sizes. Figure 8 shows a resonance step at $v_{\text {step }} \approx \Omega$ for the case of weak array/oscillator coupling, $\Lambda$ $=0.01$, in an array with $N=50$ junctions. Although not shown, the phase synchronization behavior, as measured by $r$, along the step is qualitatively similar to that shown in Fig. 4(b) for $N=10$. Figure 9 shows that it is possible to use the multistable nature of the junctions, for bias currents less than unity but greater than the retrapping current, to bias a controlled number of junction, $N_{a}$, on the resonance step. The figure shows steps for $N_{a}=50$ down to $N_{a}=25$ in increments of 5. The dashed lines show the expected location of the steps based on Eq. (61).

\section{ANALYTIC RESULTS}

To obtain analytical results, we combine Eqs. (38)-(41) to form two second-order differential equations. The results are

$$
\begin{aligned}
& \beta_{c} \frac{d^{2} \gamma_{k}}{d \tau^{2}}+i_{c k} \frac{d \gamma_{k}}{d \tau}+i_{c k} \sin \gamma_{k} \\
& =\frac{\tilde{C}}{1+\tilde{C}}\left[i_{B}+\frac{2 \sqrt{\Lambda}}{\Omega^{2}} \frac{d^{2} \tilde{a}_{I}}{d \tau^{2}}+\frac{1}{N \tilde{C}} \sum_{j=1}^{N} i_{c j}\left(\sin \gamma_{j}+\frac{d \gamma_{j}}{d \tau}\right)\right] \\
& \times(k=1, \ldots, N), \\
& \frac{d^{2} \tilde{a}_{I}}{d \tau^{2}}+\Omega^{2} \tilde{a}_{I}=-\frac{\Omega^{2} \sqrt{\Lambda}}{2} \sum_{j=1}^{N} \gamma_{j}
\end{aligned}
$$

where $\widetilde{C} \equiv\langle C\rangle /\left(N C_{o}\right)$. To obtain Eq. (46), we have substituted for the junction charge variable $\tilde{n}_{k}$ in terms of the Jo- 

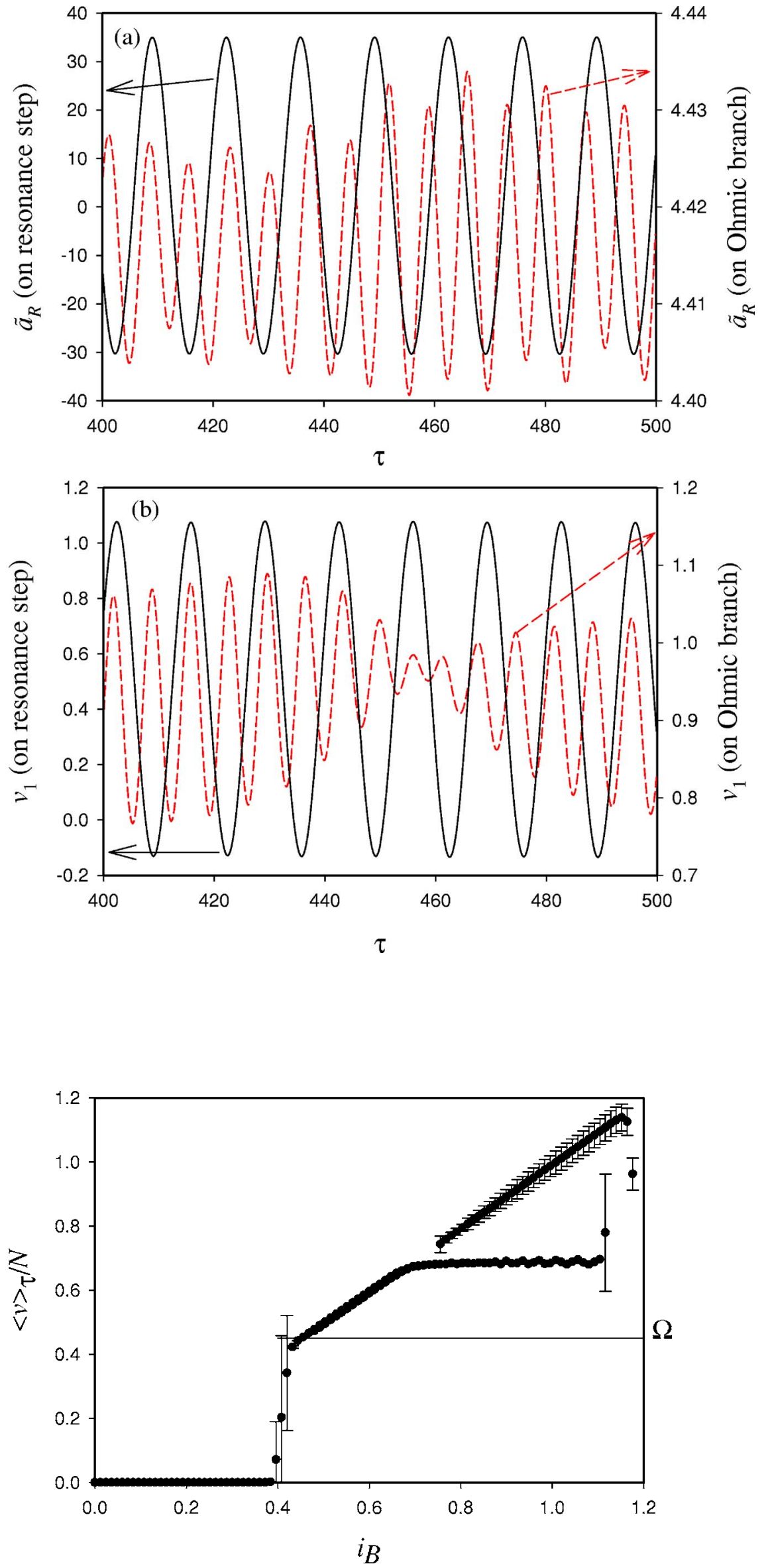

FIG. 5. (Color online) (a) Time dependence of $\tilde{a}_{R}$, which is proportional to the NEM oscillator's displacement from equilibrium, for $i_{B}=0.9$ both on the resonance step (full curve) and on the Ohmic branch (dashed curve). Note the different scales on the two vertical axes. The junction and oscillator parameters are the same as in Fig. 3. (b) Time dependence of $v_{1}$, the voltage across junction 1 , for $i_{B}=0.9$ both on the resonance step (full curve) and on the Ohmic branch (dashed curve). In both parts (a) and (b) the resonant behavior on the step is evident.
FIG. 6. $I-V$ curve for a serial array of $N=10$ underdamped junctions coupled to a NEM oscillator. Junction critical currents are assigned nonrandomly with $\Delta=0.05$ and $\beta_{c}=10$. The NEM's natural frequency is $\Omega=0.45$, and the coupling strength between the array and the NEM is $\Lambda$ $=3$. The ratio of the average junction capacitance to the capacitance of the NEM is $\langle C\rangle / C_{o}=1$. A step due to resonance between the junctions and the NEM is evident at a voltage considerably greater than $\Omega$, namely $\langle v\rangle_{\tau} / N=0.6851 \pm 0.0009$. The retrapping current is approximately equal to $\Omega$. 


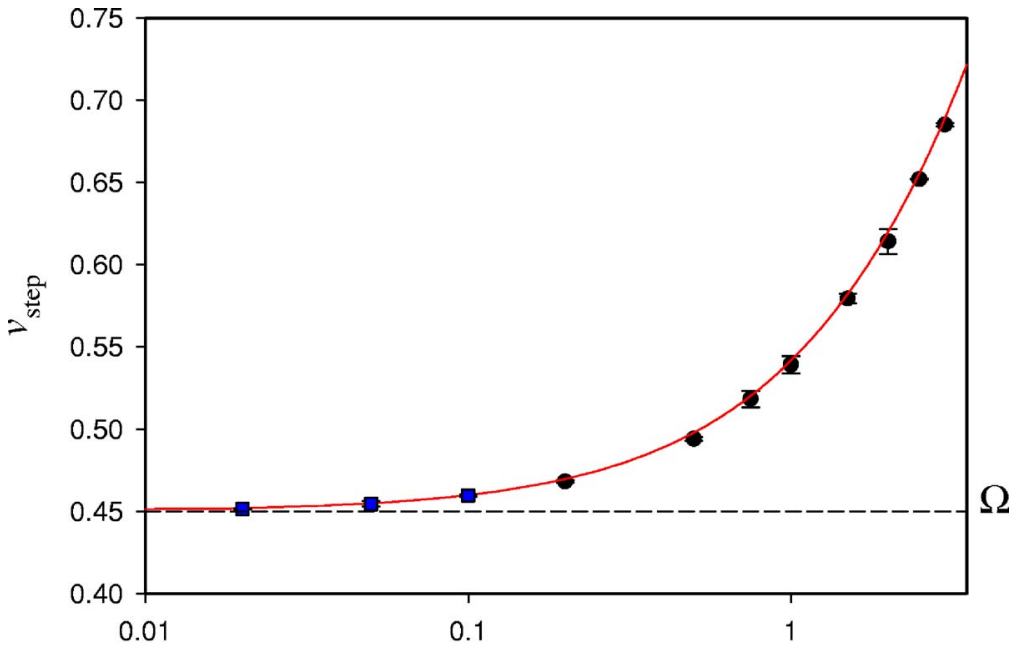

$\Lambda$
FIG. 7. (Color online) Location of the voltage step as a function of the coupling strength $\Lambda$ between a serial array $\left(N=10, \beta_{c}=10\right)$ and NEM oscillator. The NEM's natural frequency is $\Omega$ $=0.45$ and $\langle C\rangle / C_{o}=10$. Junction critical currents are assigned nonrandomly with $\Delta=0.05$. Because the resonance step could not be seen for the nonuniform array at very small values of the coupling $\Lambda<0.1$, the results for $v_{\text {step }}$ for $\Lambda \leqslant 0.1$ were obtained for the array with uniform junctions (blue symbols on graph). The dashed horizontal line at $v_{\text {step }}=\Omega$ is shown for reference. The solid line is an analytic result for $v_{\text {step }}$ given by Eq. (61). sephson phase $\gamma_{k}$ and the oscillator variable $\tilde{a}_{I}$ [see Eqs. (38) and (39)]. Equation (47) represents, as expected, a simple harmonic oscillator perturbed by the coupling (of strength $\Lambda$ ) to the array. Equation (46) shows how an independent series $\mathrm{JJ}$ array biased by a current source is affected by the NEM oscillator. In the special case of the array decoupled from the NEM, $\Lambda \rightarrow 0$ and $\widetilde{C} \rightarrow \infty$. Then the second and third terms inside the square brackets on the right-hand side of Eq. (46) will vanish and only the factor of $i_{B}$ will remain. In this decoupled limit, the resulting form of Eq. (46) is expected from the RCSJ model for a serial array of underdamped junctions coupled only to a current source. It is interesting to compare Eqs. (46) and (47) with the analogous expressions for the case of a serial array in an electromagnetic resonant cavity, ${ }^{32}$ in which case the junctions are coupled to the cavity via each junction's gauge-invariant phase difference. In the case of the resonant cavity, the $d^{2} \tilde{a}_{I} / d \tau^{2}$ term in Eq. (46) is replaced by a term proportional to $d^{2} \tilde{a}_{R} / d \tau^{2}$, and there is nothing analogous to the last term on the right-hand side. Also, for an array coupled to a resonant cavity, the term on the right-hand side of Eq. (47) is proportional to a sum over $d^{2} \gamma_{j} / d \tau^{2}$ instead of $\gamma_{j}$

Some progress in understanding the consequences of Eqs. (46) and (47) can be made by noting that on the phase-locked part of the resonance step [see Fig. 4(b)] the junctions can be thought of as $N$ underdamped, driven pendula whirling in

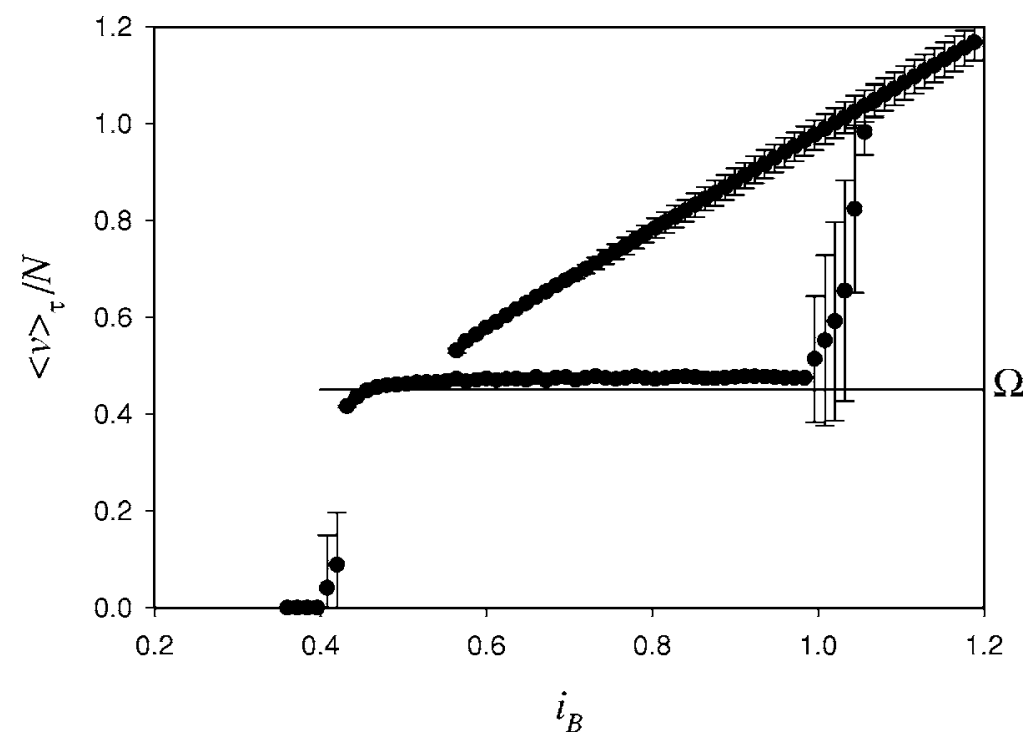

FIG. 8. $I-V$ curve for a serial array of $N=50$ underdamped junctions coupled to a NEM oscillator. Junction critical currents are assigned nonrandomly with $\Delta=0.05$ and $\beta_{c}=10$. The NEM's natural frequency, scaled by the Josephson frequency, is $\Omega=\omega_{o} / \omega_{J}=0.45$, and the coupling strength between the array and the NEM oscillator is $\Lambda=0.01$. The ratio of the average junction capacitance to the capacitance of the NEM is $\langle C\rangle / C_{o}=50$. A step due to resonance between the junctions and the NEM is evident at a voltage only slightly greater than $\Omega$, namely $\langle v\rangle_{\tau} / N=0.5476 \pm 0.0187$. The error bars on the symbols represent the standard deviation of the mean of the time-averaged $\mathrm{JJ}$ voltages. As the bias current is decreased from its starting value of $i_{B}=1.2$, the error bars become smaller than the symbol size, signaling approximate frequency synchronization of the array. 


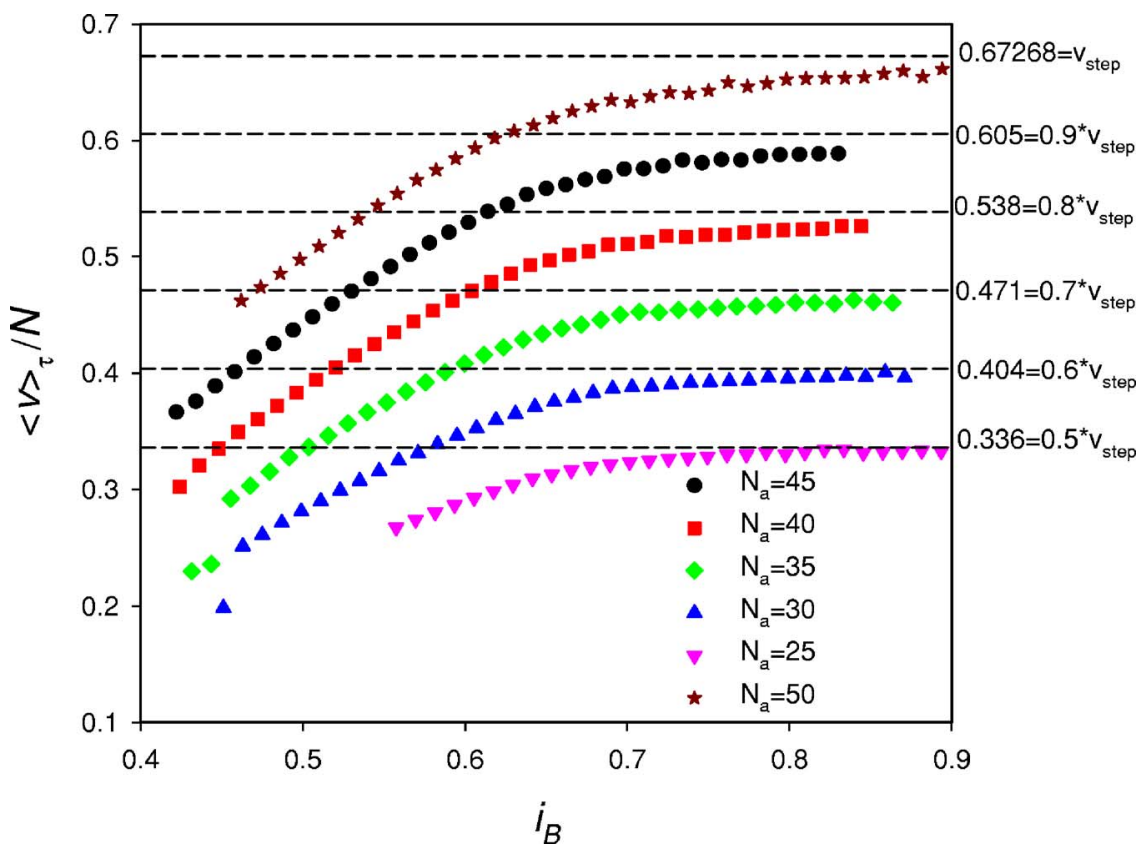

FIG. 9. (Color online) $I-V$ curve for a serial array of $N=50$ underdamped junctions coupled to a NEM oscillator showing different numbers of junctions, $N_{a}$, biased on the resonance step. $N-N_{a}$ junctions are in the zero-voltage state. The curves represent $N_{a}=25,30,35$, 40, 45, and 50. Junction critical currents were uniform and $\beta_{c}=10$. The NEM's natural frequency, scaled by the Josephson frequency, is $\Omega=\omega_{o} / \omega_{J}=0.45$, and the coupling strength between the array and the NEM oscillator is $\Lambda=0.1$. The ratio of the average junction capacitance to the capacitance of the NEM is $\langle C\rangle / C_{o}=50$. Each curve was produced by increasing the bias current from a starting value between 0.41 to 0.45 . The dotted lines represent the expected location of the step, $\left(N_{a} / N\right) v_{\text {step }}$, where the value of $v_{\text {step }}$ is obtained from Eq. (61).

phase with the same angular velocity. So the phase-locked behavior of the junctions can be represented by the following Ansatz for the Josephson phase difference (the same for all $N$ junctions):

$$
\gamma=\phi+v_{\text {step }} \tau+A \sin \left(v_{\text {step }} \tau+\delta\right),
$$

where $\gamma, v_{\text {step }}, A$, and $\delta$ are all constants. Equation (48) describes a linearly growing Josephson phase with a sinusoidal oscillation of amplitude $A$ superimposed. Alternatively, it describes the angular displacement of a pendulum whirling with a nearly constant angular velocity perturbed by sinusoidal variation due to a gravitational torque. The derivatives of Eq. (48) (the angular velocity and acceleration of the pendulum) are

$$
\begin{gathered}
\frac{d \gamma}{d \tau}=v_{\text {step }}+v_{\text {step }} A \cos \left(v_{\text {step }} \tau+\delta\right), \\
\frac{d^{2} \gamma}{d \tau^{2}}=-v_{\text {step }}^{2} A \sin \left(v_{\text {step }} \tau+\delta\right)
\end{gathered}
$$

Note that location of the voltage step is given by the average of Eq. (49), i.e., $\langle d \gamma / d \tau\rangle_{\tau}=v_{\text {step }}$, where the notation $\langle X\rangle_{\tau}$ denotes a time average of the quantity $X$. A main goal of this section will be to derive an analytic expression for $v_{\text {step }}$. To justify Eq. (48), we have fit numerical solutions to Eqs. (38)-(41) in the phase-locked region of a resonant step with expressions of the form of Eqs. (48)-(50). The results are shown in Fig. 10, where the excellent agreement between the numerical results (solid lines) and the fit (symbols) is evident.

Using Eqs. (48)-(50) it is possible to solve for the behavior of the NEM oscillator via Eq. (47). We get a general result of

$$
\begin{aligned}
\tilde{a}_{I}(\tau)= & \alpha \sin \Omega \tau+\beta \cos \Omega \tau-\frac{N \phi \sqrt{\Lambda}}{2}-\frac{N v_{\text {step }} \sqrt{\Lambda}}{2} \tau \\
& +\frac{N \Omega^{2} \sqrt{\Lambda}}{2\left(v_{\text {step }}^{2}-\Omega^{2}\right)} A \sin \left(v_{\text {step }} \tau+\delta\right),
\end{aligned}
$$

where $\alpha$ and $\beta$ are arbitrary constants. In the stable region of the step, however, the oscillator is in a strong resonance with the junctions, and both are exhibiting sinusoidal behavior with the same angular frequency, $v_{\text {step }}$. The last term in Eq. (51), the sinusoidal term with the resonant amplitude, should dominate over the other sinusoidal terms with angular frequency $\Omega$. We can thus approximate Eq. (51) with

$$
\begin{aligned}
\tilde{a}_{I}(\tau)= & -\frac{N \phi \sqrt{\Lambda}}{2}-\frac{N v_{\text {step }} \sqrt{\Lambda}}{2} \tau+\frac{N \Omega^{2} \sqrt{\Lambda}}{2\left(v_{\text {step }}^{2}-\Omega^{2}\right)} \\
& \times A \sin \left(v_{\text {step }} \tau+\delta\right) .
\end{aligned}
$$

Figure 11 shows $\tilde{a}_{I}+\left(N v_{\text {step }} \sqrt{\Lambda} \tau\right) / 2$ from the numerical solution of Eqs. (38)-(41), where the numerical value of $v_{\text {step }}$ was taken from the fit in Fig. 10(a). The fit to the results are designated by the crosshair symbols. The agreement is excellent.

Using Eq. (52), we substitute $d^{2} \widetilde{a}_{I} / d \tau^{2}$ into Eq. (46), and letting $i_{c k}=1$ for all junctions (which should be reasonable 

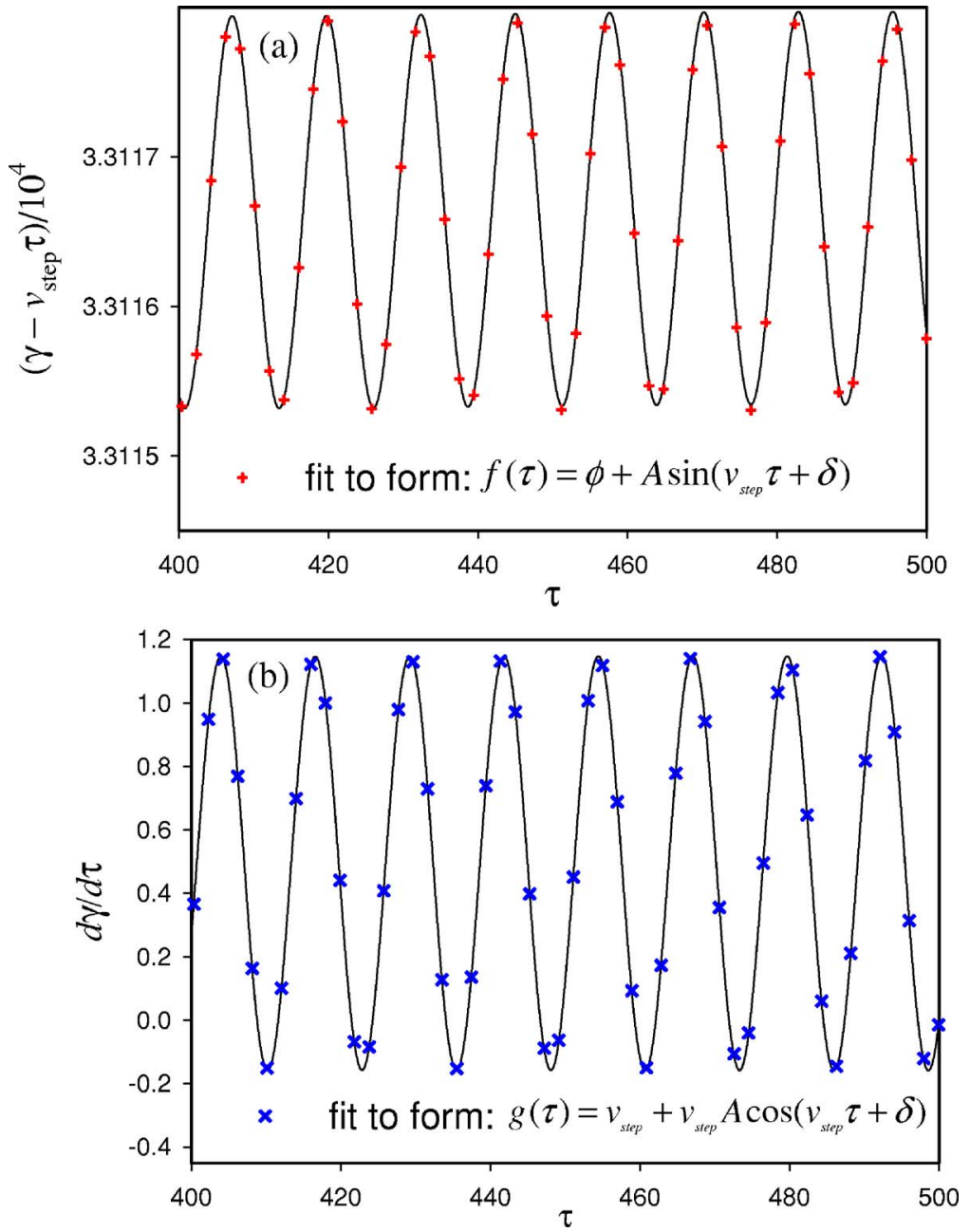

FIG. 10. (Color online) Numerical solutions of Eqs. (38)-(41) for $\gamma(\tau)$ and $d \gamma / d \tau$ versus time and fits to these numerical results. Parameters are $N=10, \beta_{c}=10,\langle C\rangle / C_{o}=1, \Lambda=0.5$, $\Omega=0.45$. For simplicity, the junction critical currents were taken to be uniform. (a) Josephson phase $\gamma$ with the linearly growing term, $v_{\text {step }} \tau$, subtracted out, leaving oscillatory behavior. The crosshairs represent a regression fit to a function $f(\tau)=\phi+A \sin \left(v_{\text {step }} \tau+\delta\right)$. From the fit, we find $A=1.3051$ and $v_{\text {step }}=0.4978$. (b) The voltage across an individual junction, $d \gamma / d \tau$. The " $\times$ " symbols represent a regression fit to a function $g(\tau)=v_{\text {step }}+v_{\text {step }} A \cos \left(v_{\text {step }} \tau+\delta\right)$. As a consistency check, note that the amplitude of $d \gamma / d \tau$ can be estimated from the graph and is in good agreement with the numerical value of $v_{\text {step }} A$ as specified by Eq. (49), where the values of $v_{\text {step }}$ and $A$ are taken from the fit in part (a). for small amounts of critical-current disorder), we get the expression

$$
\begin{aligned}
& v_{\text {step }}^{2}\left(\frac{N \Lambda}{\left(v_{\text {step }}^{2}-\Omega^{2}\right)}-\kappa\right) A \sin X+v_{\text {step }} A \cos X+v_{\text {step }} \\
& \quad+\sin \left(\phi+v_{\text {step }} \tau+A \sin X\right)=i_{B},
\end{aligned}
$$

where $\kappa \equiv \beta_{c}(1+\widetilde{C}) / \widetilde{C}$ and $X \equiv v_{\text {step }} \tau+\delta$. Making use of the

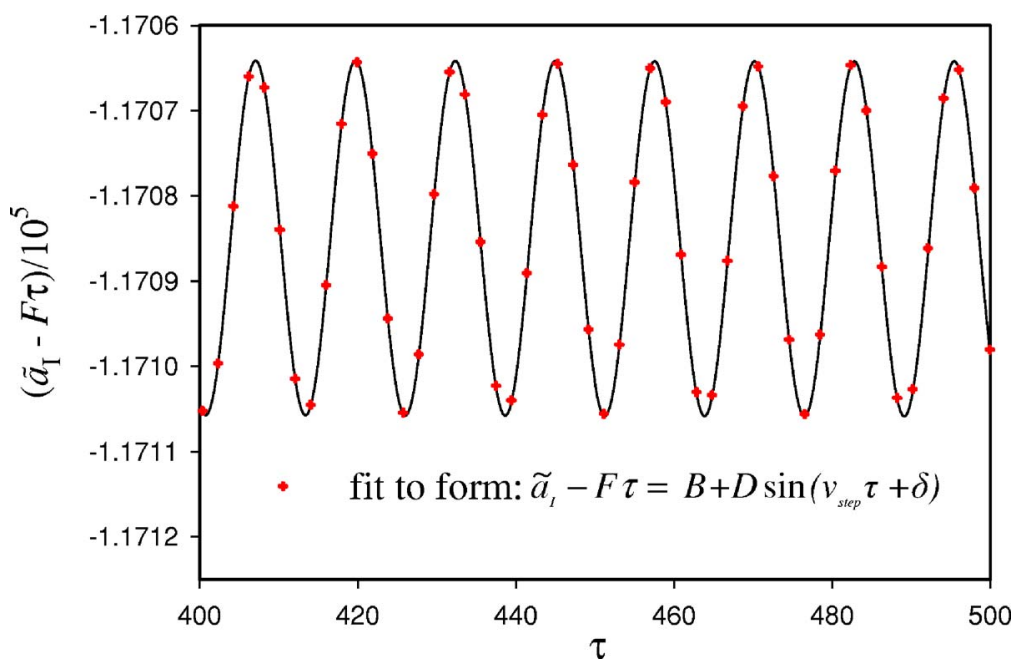

well-known Bessel function expansion for the sine of a sine or cosine, we can write

$$
\begin{aligned}
\sin (\phi+ & \left.v_{\text {step }} \tau+A \sin X\right) \\
= & J_{0}(A) \sin (X+\phi-\delta)+\sum_{n=1}^{\infty} J_{n}(A) \\
& \times\left\{\sin [(n+1) X+(\phi-\delta)]-(-1)^{n}\right.
\end{aligned}
$$

FIG. 11. (Color online) Numerical solution for $\tilde{a}_{I}$ versus time. For convenience, the linearly growing (in time) contribution is subtracted out, and the vertical axis is scaled by a factor of $10^{5}$. Parameters are $N=10, \beta_{c}=10,\langle C\rangle / C_{o}=1, \Lambda$ $=0.5, \Omega=0.45$. For simplicity, the junction critical currents were taken to be uniform. The numerical value of the constant $F$ was determined from the fact that $F=-N v_{\text {step }} \sqrt{\Lambda} / 2$. From the fit, we find $D=20.6987$, which agrees with the expression $D=\left\{N \Omega^{2} \sqrt{\Lambda} /\left[2\left(v_{\text {step }}^{2}-\Omega^{2}\right)\right]\right\} A$ [see Eq. (51)], if we use the value $A=1.3051$ from Fig. 10. 


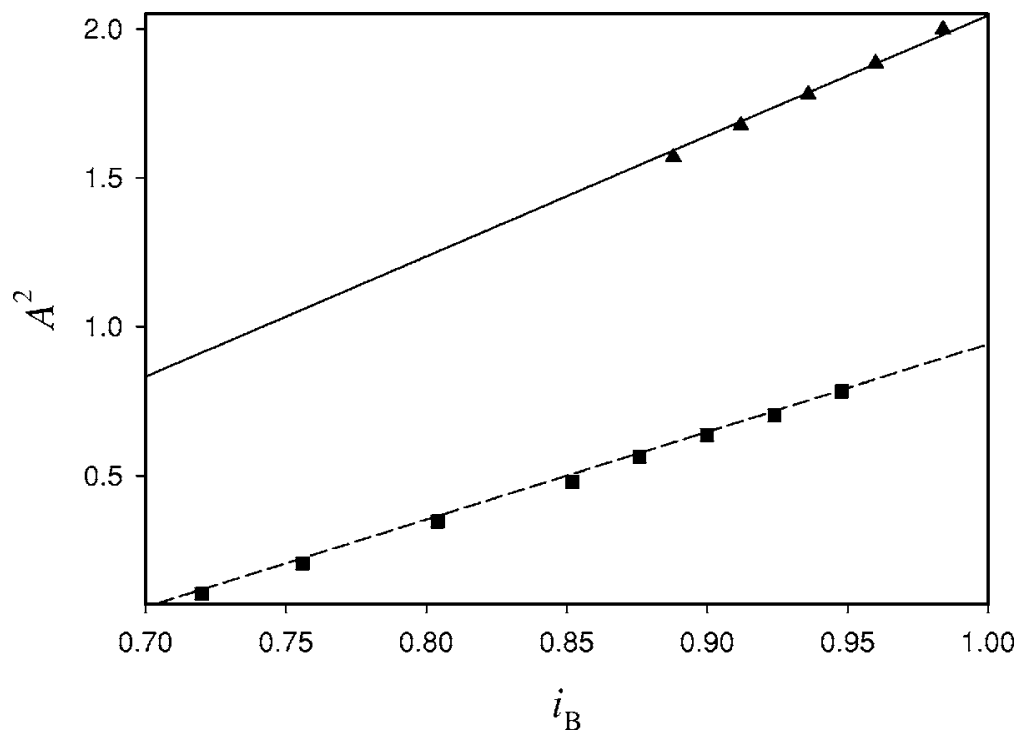

FIG. 12. Square of the amplitude of the sinusoidal contribution to the Josephson phase versus the dc bias current. Parameters are $N=10, \beta_{c}$ $=10,\langle C\rangle / C_{o}=1$, and $\Omega=0.45$. The triangle symbols denote numerical results for the squared amplitude of the Josephson phase oscillations, denoted by $A^{2}$, for a coupling $\Lambda=0.5$. The solid line is the result $A^{2}=2\left(i_{B} / v_{\text {step }}-1\right)$ for $v_{\text {step }}=0.4945$ versus $i_{B}$. The square symbols denote numerical results for $A^{2}$ for $\Lambda=3$. The dotted line is the result $A^{2}=2\left(i_{B} / v_{\text {step }}-1\right)$ for $v_{\text {step }}=0.68$. The numerical results are obtained only for those applied currents on the step where phase locking occurs.

$$
\times \sin [(n-1) X-(\phi-\delta)]\},
$$

where $J_{n}(A)$ is the Bessel function of order $n$. Substituting Eq. (54) into Eq. (53), we get an expression of the form

$$
\begin{aligned}
v_{\text {step }} & -i_{B}-J_{1}(A) \sin (\phi-\delta)+\sin X\left[v_{\text {step }}^{2}\left(\frac{N \Lambda}{\left(v_{\text {step }}^{2}-\Omega^{2}\right)}-\kappa\right) A\right. \\
& \left.+J_{0}(A) \cos (\phi-\delta)-J_{2}(A) \cos (\phi-\delta)\right]+\cos X\left[v_{\text {step }} A\right. \\
& \left.+J_{0}(A) \sin (\phi-\delta)+J_{2}(A) \sin (\phi-\delta)\right]+\cdots=0
\end{aligned}
$$

where the $\cdots$ represents all the higher harmonic terms from the expansion in Eq. (54). Using the principle of harmonic balance, we equate each multiple of $\sin X, \cos X$, and the constant term to 0 . The results are the following expressions:

$$
\begin{gathered}
i_{B}=v_{\text {step }}-J_{1}(A) \sin (\phi-\delta), \\
\sin (\phi-\delta)=-\frac{v_{\text {step }} A}{J_{0}(A)+J_{2}(A)}, \\
\cos (\phi-\delta)=\frac{v_{\text {step }}^{2}\left(\frac{N \Lambda}{\left(v_{\text {step }}^{2}-\Omega^{2}\right)}-\kappa\right) A}{J_{2}(A)-J_{0}(A) .} .
\end{gathered}
$$

Substituting Eq. (57) into Eq. (56) and making use of the identity $J_{n+1}(x)+J_{n-1}(x)=(2 n / x) J_{n}(x)$ leads to an expression for the amplitude of the sinusoidal oscillation of the Josephson phases [refer to Eq. (48)]

$$
A=\sqrt{2\left(\frac{i_{B}}{v_{\text {step }}}-1\right)} .
$$

The validity of Eq. (59) can be checked by solving Eqs. (38)-(41) numerically and fitting the results to Eqs. (48)-(50) to determine a value for $A$. In Fig. 12, we plot $A^{2}$ versus $i_{B}$ along a given step $v_{\text {step }}$ for two different values of the coupling strength $\Lambda$. We find that Eq. (59) is accurate over that region of the step that exhibits phase locking (i.e., where $r \approx 1$ ), which is just where our Ansatz, Eq. (48), should be valid.

Since $\sin ^{2}(\phi-\delta)+\cos ^{2}(\phi-\delta)=1$, we can combine Eqs. (57) and (58)

$$
\begin{gathered}
2 v_{\text {step }}^{2}\left(\frac{i_{B}}{v_{\text {step }}}-1\right)\left[\frac{1}{\left[J_{0}(A)+J_{2}(A)\right]^{2}}\right. \\
\left.+\frac{\left.\left(\frac{N \Lambda}{\left(v_{\text {step }}^{2}-\Omega^{2}\right)}-\kappa\right)^{2}\right]}{\left[J_{0}(A)-J_{2}(A)\right]^{2}}\right]=1,
\end{gathered}
$$

where Eq. (59) was used. One cannot solve Eq. (60) analytically, but MATHEMATICA can be used to find $v_{\text {step }}$ as a function of the variable $i_{B}$. We find that the solution for $v_{\text {step }}$ describing the resonance step is well approximated by choosing $\cos (\phi-\delta)=0$. From Eq. (58), this results in an expression that can be solved for $v_{\text {step }}$

$$
v_{\text {step }}=\sqrt{\Omega^{2}+\frac{N \Lambda}{\kappa}}=\sqrt{\Omega^{2}+\frac{\Lambda\left(\langle C\rangle / C_{o}\right)}{\beta_{c}\left(1+\frac{\langle C\rangle}{N C_{o}}\right)}} .
$$

This result for the step location is shown as a solid (red) curve in Fig. 7, where it is seen to fit well the numerical results for $v_{\text {step }}$. Equation (61) shows that as the array and oscillator coupling becomes weak, the voltage step coincides with the oscillator's natural frequency, i.e., as $\Lambda \rightarrow 0$, then $v_{\text {step }} \rightarrow \Omega$. That is, strong coupling shifts the step location to a higher voltage.

Equation (61) shows several interesting features. As the array size increases for a fixed value of the ratio $\langle C\rangle / C_{o}$, the term in the denominator proportional to $1 / N$ becomes less important. So in the limit of a large array ("large" means $N$ $\gtrsim 10\langle C\rangle / C_{o}$ ) the importance of the coupling term in Eq. (61) depends on the McCumber parameter $\beta_{c}$ of the junctions as well as on the ratio of the average junction capacitance to the capacitance of the NEM oscillator, $\langle C\rangle / C_{o}$. In fact, we see 
that increasing the junctions' McCumber parameter makes the second term inside the square root less effective at shifting the step location upward from $\Omega$. By contrast, increasing the ratio $\langle C\rangle / C_{o}$ does just the opposite, i.e., it makes the coupling term more effective at shifting the step voltage upward from $\Omega$. This behavior could provide, via the fabrication process, a means to control the location of the resonance step. From a technological standpoint, such control could be important.

In both experimental and theoretical studies of resonant phase-locking of JJ arrays, scaling of the energy output with the square of the number of active junctions $N_{a}$ has been emphasized as evidence for coherent behavior. ${ }^{31,32}$ Using Eq. (48), we can show analytically that in the phase-locked region of a resonance step the time-averaged energy of the NEM oscillator does indeed scale like $N_{a}^{2}$. Before converting to dimensionless units, the time-averaged oscillator energy is

$$
E_{o}=\hbar \omega_{o}\left(\left\langle a^{\dagger} a\right\rangle_{t}+\frac{1}{2}\right)=\hbar \omega_{o}\left(\left\langle a_{R}^{2}+a_{I}^{2}\right\rangle_{t}+\frac{1}{2}\right),
$$

where we have used the fact that $a \equiv a_{R}+i a_{I}$ and $a^{\dagger} \equiv a_{R}$ $-i a_{I}$. Subtracting off the zero-point energy, and writing Eq. (62) in terms of dimensionless quantities, we get

$$
\tilde{E}_{o}=\left\langle\widetilde{a}_{R}^{2}+\widetilde{a}_{I}^{2}\right\rangle_{\tau},
$$

where $\widetilde{E}_{o} \equiv\left(E_{o}-\frac{1}{2} \hbar \omega_{o}\right) /\left(\hbar\left\langle I_{c}\right\rangle / e\right)$.

Suppose $N_{a}$ junctions are active, i.e., biased on the resonance step, while the remaining $N-N_{a}$ junctions are in the zero-voltage state. Equation (52) would then be changed only in that the multiplicative factor $N$ would be changed to $N_{a}$. Using Eqs. (41) and (52), we find an expression for $\tilde{a}_{R}$ when $N_{a}$ junctions are active

$$
\widetilde{a}_{R}=\frac{N_{a} v_{\text {step }}}{2 \Omega}\left[\sqrt{\Lambda}-\frac{\Omega^{2} \sqrt{\Lambda}}{v_{\text {step }}^{2}-\Omega^{2}} A \cos \left(v_{\text {step }} \tau+\delta\right)\right] .
$$

Finally, we substitute Eqs. (52) and (64) into Eq. (63) and perform the time average. Using that $\langle\sin X\rangle_{\tau}=\langle\cos X\rangle_{\tau}=0$ and $\left\langle\sin ^{2} X\right\rangle_{\tau}=\left\langle\cos ^{2} X\right\rangle_{\tau}=\frac{1}{2}$, where the time average is over an integer number of cycles, we get the result

$$
\widetilde{E}_{o}=\frac{N_{a}^{2} \Lambda}{4}\left[\frac{4 \pi^{2}}{3}+2 \pi \phi+\phi^{2}+\frac{v_{\text {step }}^{2}}{\Omega^{2}}\left(1+\frac{A^{2}}{2} \frac{\left(1+\frac{\Omega^{2}}{v_{\text {step }}^{2}}\right)}{\left(1-\frac{v_{\text {step }}^{2}}{\Omega^{2}}\right)^{2}}\right)\right] \text {, }
$$

where $v_{\text {step }}$ and $A$ are given by Eqs. (59) and (61), respectively. The dependence of the resonator energy on the square of the number of active junctions is evident. This result depends upon the validity of Eq. (48). If, for sufficiently small $N_{a}$, Eq. (48) no longer describes the phase behavior of the active junctions on the step, then our result for the resonator energy would not hold. A consequence of the failure of Eq.
(48) could be that there is a threshold number of active junctions needed before the onset of coherence (i.e., $\widetilde{E}_{o} \propto N_{a}^{2}$ ) is observed.

\section{DISCUSSION AND CONCLUSION}

The growing sophistication in the fabrication of NEMs, whether based on materials such as aluminum nitride ${ }^{34}$ or carbon nanotubes, ${ }^{39}$ means that the technology of coupling very high- $Q$ oscillators to JJ arrays is becoming mature. Such maturity means it should be possible to fabricate a serial or two-dimensional JJ array with an on-chip NEM device. It would be of interest in the field of millimeter-wave production to see if a strong coupling between the junctions and the NEM could result in ac power outputs comparable or even larger than those seen in photon-induced coupling schemes. ${ }^{29,31}$ Based on Eq. (12) we see that the coupling strength $\lambda$ between the NEM and the junctions could in principle be controlled by the NEM oscillator dimensions, such as its diameter $b$. In this paper, we have shown theoretically that a serial array of nonuniform JJs coupled piezoelectrically to an AlN dilatational oscillator can result in phaselocking of the junctions.

Our approach, similar to that in Ref. 32, is based upon a semiclassical solution to a set of coupled differential equations that were generated from the quantum Heisenberg equations for the Hamiltonian $H=H_{J}+H_{N E M}+H_{\text {diss }}+H_{\text {int }}$, where the terms in the Hamiltonian are given by Eqs. (22)-(26). The phase-locked state of the junctions is signaled by a step in the $I-V$ curve. Interestingly, we find (neutrally) stable phase locking only at the top and bottom of the resonance step. It would be useful to determine if the unstable solution in the middle of the voltage step is a result of the particular form of coupling used, $H_{\text {int }}$, in this geometry. Different NEM geometries with different physics underlying the coupling to the junctions should be studied as well.

Another consequence of our model is that the energy of the oscillator growing quadratically with the number of junctions biased on the resonance step, a result that was also seen in the case of photon-induced coupling. ${ }^{32}$

The model discussed in this paper could be a feasible beginning for a fully quantum mechanical description of small JJs coupled to a NEM oscillator. ${ }^{40}$ Such a study would not be limited to the case of large phonon number or a large number of charges on the junctions. One goal of such a study would be to see if a resonance could result in an entangled state of many junctions and the oscillator.

\section{ACKNOWLEDGMENTS}

B.R.T. wishes to thank the hospitality of physics department at Ohio State University and Ohio Wesleyan University for support. S.N. acknowledges Ohio Wesleyan for support for summer research. B.R.T. acknowledges the Ohio Supercomputing Center for a grant of time. D.S. acknowledges the Ohio Supercomputing Center for a grant of time and NSF Grant No. DMR04-13395 for support. 
*Electronic address: brtrees@ owu.edu

${ }^{1}$ M. P. Blencowe, Phys. Rep. 395, 159 (2004).

${ }^{2}$ R. G. Knobel and A. N. Cleland, Nature (London) 424, 291 (2003).

${ }^{3}$ M. D. LaHaye, O. Buu, B. Camarota, and K. C. Schwab, Science 304, 74 (2004).

${ }^{4}$ A. D. Armour, M. P. Blencowe, and K. C. Schwab, Phys. Rev. Lett. 88, 148301 (2002).

${ }^{5}$ X. Zou and W. Mathis, Phys. Lett. A 324, 484 (2004).

${ }^{6}$ A. N. Cleland and M. R. Geller, Phys. Rev. Lett. 93, 070501 (2004).

${ }^{7}$ K. B. Cooper, M. Steffen, R. McDermott, R. W. Simmonds, S. Oh, D. A. Hite, D. P. Pappas, and J. M. Martinis, Phys. Rev. Lett. 93, 180401 (2004).

${ }^{8}$ R. W. Simmonds, K. M. Lang, D. A. Hite, S. Nam, D. P. Pappas, and J. M. Martinis, Phys. Rev. Lett. 93, 077003 (2004).

${ }^{9}$ J. Eisert, M. B. Plenio, S. Bose, and J. Hartley, Phys. Rev. Lett. 93, 190402 (2004).

${ }^{10}$ See Ref. 1 and references therein.

${ }^{11}$ R. Ruby, P. Bradley, J. Larson, Y. Oshmyansky, and D. Figueredo, Technical Digest of the 2001 IEEE International Solid-State Circuits Conference, 2001, Piscataway, NJ (unpublished), pp. 120-121.

${ }^{12}$ See references 6 and 7 in Ref. 6.

${ }^{13}$ A. Pikovsky, M. Rosenblum, and J. Kurths, Synchronization: A Universal Concept in Nonlinear Sciences (Cambridge University Press, Cambridge, UK, 2001).

${ }^{14}$ K. Wiesenfeld, P. Colet, and S. H. Strogatz, Phys. Rev. Lett. 76, 404 (1996).

${ }^{15}$ H. S. J. van der Zant, T. P. Orlando, S. Watanabe, and S. H. Strogatz, Phys. Rev. Lett. 74, 174 (1995).

${ }^{16}$ S. Watanabe, S. H. Strogatz, H. S. J. van der Zant, and T. P. Orlando, Phys. Rev. Lett. 74, 379 (1995).

${ }^{17}$ P. A. A Booi and S. P. Benz, Appl. Phys. Lett. 68, 3799 (1996).

${ }^{18}$ S. Han, B. Bi, W. Zhang, and J. E. Lukens, Appl. Phys. Lett. 64, 1424 (1994).

${ }^{19}$ V. K. Kaplunenko, J. Mygind, N. F. Pedersen, and A. V. Ustinov, J. Appl. Phys. 73, 2019 (1993).

${ }^{20}$ K. Wan, A. K. Jain, and J. E. Lukens, Appl. Phys. Lett. 54, 1805 (1989).
${ }^{21}$ P. Hadley, M. R. Beasley, and K. Wiesenfeld, Phys. Rev. B 38, 8712 (1988).

${ }^{22}$ M. Octavio, C. B. Whan, and C. J. Lobb, Appl. Phys. Lett. 60, 766 (1992).

${ }^{23}$ K. Wiesenfeld, S. P. Benz, and P. A. A. Booi, J. Appl. Phys. 76, 3835 (1994).

${ }^{24}$ Y. Braiman, W. L. Ditto, K. Wiesenfeld, and M. L. Spano, Phys. Lett. A 206, 54 (1995).

${ }^{25}$ M. Darula, S. Beuven, M. Siegel, A. Darulova, and P. Seidel, Appl. Phys. Lett. 67, 1618 (1995).

${ }^{26}$ C. B. Whan, A. B. Cawthorne, and C. J. Lobb, Phys. Rev. B 53, 12340 (1996).

${ }^{27}$ G. Filatrella, N. F. Pedersen, and K. Wiesenfeld, Appl. Phys. Lett. 72, 1107 (1998).

${ }^{28}$ G. Filatrella, N. F. Pedersen, and K. Wiesenfeld, Phys. Rev. E 61, 2513 (2000).

${ }^{29}$ B. Vasili'c, S. V. Shitov, C. J. Lobb, and P. Barbara, Appl. Phys. Lett. 78, 1137 (2001).

${ }^{30}$ G. Filatrella, N. F. Pedersen, C. J. Lobb, and P. Barbara, Eur. Phys. J. B 34, 3 (2003).

${ }^{31}$ P. Barbara, A. B. Cawthorne, S. V. Shitov, and C. J. Lobb, Phys. Rev. Lett. 82, 1963 (1999).

${ }^{32}$ E. Almaas and D. Stroud, Phys. Rev. B 65, 134502 (2002).

${ }^{33}$ E. Almaas and D. Stroud, Phys. Rev. B 67, 064511 (2003).

${ }^{34}$ A. N. Cleland, M. Pophristic, and I. Ferguson, Appl. Phys. Lett. 79, 2070 (2001).

${ }^{35}$ A. O. Caldeira and A. J. Leggett, Ann. Phys. (N.Y.) 149, 374 (1983).

${ }^{36}$ Ch. Helm, Ch. Preis, F. Forsthofer, J. Keller, K. Schlenga, R. Kleiner, and P. Müller, Phys. Rev. Lett. 79, 737 (1997).

${ }^{37}$ S. Chakravarty, G-L. Ingold, S. Kivelson, and A. Luther, Phys. Rev. Lett. 56, 2303 (1986).

${ }^{38}$ For a discussion of a standard numerical technique for calculating Floquet multipliers, see S. Nichols and K. Wiesenfeld, Phys. Rev. A 45, 8430 (1992).

${ }^{39}$ S. B. Legoas, V. R. Coluci, S. F. Braga, P. Z. Coura, S. O. Dantas, and D. S. Galvão, Nanotechnology 15, S184 (2004).

${ }^{40}$ For the case of photon-induced coupling, see W. A. Al-Saidi and D. Stroud, Phys. Rev. B 65, 014512 (2002). 